Rev Inv Vet Perú 2009; 20 (2): 213-220

\title{
EFECTO DEL TRATAMIENTO DE LA CAMA CON UN ALUMINOSILICATO EN POLLOS DE CARNE
}

\author{
EfFect of Treatment of Broiler LitTer with Aluminosilicate
}

\author{
Melissa Pizarro R. ${ }^{1}$, Eliana Icochea D. ${ }^{1,2}$, Pablo Reyna S. ${ }^{1}$ y Néstor Falcón P. ${ }^{3,4}$
}

\section{RESUMEN}

El estudio evaluó el efecto del tratamiento de la cama con un aluminosilicato activado sobre los parámetros productivos en pollos de carne. Se utilizaron 400 pollos de carne de ambos sexos de la línea Ross 308. La mitad se crió sobre cama tratada con aluminosilicato y la otra mitad sobre cama no tratada hasta los 44 días de edad. Se analizó los datos productivos de las aves, niveles de amoniaco y $\mathrm{pH}$ atmosférico del gallinero, y $\mathrm{pH}$ y porcentaje de humedad de la cama. También se evaluó la presencia de pododermatitis y lesiones a nivel de la pechuga. La ganancia de peso, el índice de conversión alimenticia y el índice de eficiencia productivo europeo fueron estadísticamente similares entre los dos grupos. No se evidenció lesiones a nivel de la pechuga y el grado de lesiones a nivel de las patas fue similar en ambos grupos. El nivel de amoniaco, $\mathrm{pH}$ atmosférico y $\mathrm{pH}$ de la cama fue mayor en el grupo de aves criadas sobre cama no tratada demostrando que el tratamiento de la cama con aluminosilicato fue efectivo en controlar los niveles de amoniaco, aunque no se llegó a reflejar en una mejora del los niveles productivos de las aves.

Palabras clave: tratamiento, cama, aluminosilicato, amoniaco, $\mathrm{pH}$, humedad

\section{Abstract}

The present study aimed to evaluate the effect of treating broiler litter with active aluminosilicate on productive parameters of broiler chickens. Four hundred broilers Ross 308 of both sexes were reared for 44 days. Half of them were reared on litter treated with aluminosilicate and the other half on untreated litter. Productive performance of chicks and ammonia concentrations, atmospheric ph, litter ph, and moisture ratio of the litter were evaluated on a weekly basis. Additionally, lesions in breast and foot pads were also evaluated. Body weight gain, feed conversion index, and productive efficiency index were statistically similar between the two groups. No breast lesions were observed and the degree of lesions in foot pads was similar in the two groups. The ammonia

\footnotetext{
${ }^{1}$ Laboratorio de Patología Aviar, ${ }^{3}$ Laboratorio de Medicina Veterinaria Preventiva, Facultad de Medicina Veterinaria, Universidad Nacional Mayor de San Marcos, Lima

${ }^{2}$ E-mail: eliana.icochea@gmail.com

${ }^{4}$ Dirección actual: Facultad de Veterinaria y Zootecnia, Universidad Peruana Cayetano Heredia, Lima
} 
concentrations, atmospheric ph, and litter ph were higher in the non-treated litter group showing that the litter treatment with aluminosilicate was effective in controlling ammonia levels, despite no effect was observed in the productive performance.

Key words: treatment, litter, aluminosilicate, ammonia, $\mathrm{pH}$, moisture

\section{INTRODUCCIÓN}

La industria avícola ha pasado por muchos cambios en los últimos 25 años. Los galpones son de mayor superficie, las densidades de crianza han aumentado, la genética y la nutrición avícola han progresado exponencialmente, $\mathrm{y}$ todo esto ha permitido criar un mayor número de aves por año, $\mathrm{y}$ de mayor peso vivo en periodos más cortos. Sin embargo, esto ha traído como consecuencia que la crianza de aves exija mejores condiciones de alimentación e infraestructura a través de un control ambiental muy preciso (Donald, 1997).

El ambiente en los galpones es la combinación de factores físicos y biológicos que actúan en un complejo sistema dinámico de interacciones sociales, iluminación y condiciones medioambientales como temperatura, ventilación y presencia de gases tóxicos (Sainsbury, 2000). Los sistemas actuales de confinamiento y altas densidades de población propician problemas de contaminación ambiental en los galpones, donde el amoniaco es el gas que causa los más graves problemas (Quintana, 1999).

En la práctica avícola es frecuente la exposición de las aves a 50 ppm de amoniaco; sin embargo, en galpones con ventilación deficiente pueden alcanzar niveles de $200 \mathrm{ppm}$ (Carlile, 1984). Las emisiones de amoniaco tienen considerables efectos medioambientales por las altas concentraciones por metro cuadrado, que son comunes en la fase de mayor crecimiento de los pollos de carne (AlHomidan et al., 2003). Existen diversos estudios sobre los factores involucrados en la producción de amoniaco, pero falta conocer más sobre su impacto en la salud de las aves, especialmente en el peso corporal y en el índice de conversión alimenticia, aunque algunas investigaciones reportan, además, mayor mortalidad y menor consumo de alimento (Carlile, 1984).

Las altas concentraciones de amoniaco en los galpones pueden ocasionar queratoconjuntivitis, depresión respiratoria y daño patológico del tracto respiratorio, predisponiendo a infecciones secundarias y afectando el rendimiento. Esto puede comprometer la eficiente respuesta a la vacunación (Jodas y Hafez, 2001). Ante estos efectos negativos, es necesario conocer los factores que incrementan el nivel de amoniaco, así como determinar los métodos más efectivos de control (Al-Homidan et al., 2003), para evaluar en ambos casos su efecto sobre la mejor salud y rendimiento productivo de las aves.

En el mercado existen varios productos químicos que son usados para el control de la producción de amoniaco en cama de galpones de aves; sin embargo, no existe un producto con un efecto continuo por varios días que evite el uso de dos o más aplicaciones sin afectar los costos de producción. El presente estudio tuvo como objetivo evaluar el efecto del tratamiento de la cama con un aluminosilicato activado, sobre la calidad sanitaria y los parámetros productivos de los pollos de carne. 


\section{Materiales y Métodos}

\section{Infraestructura y Materiales}

El estudio se realizó en los ambientes de la Unidad de Experimentación del Laboratorio de Patología Aviar de la Facultad de Medicina Veterinaria de la Universidad Nacional Mayor de San Marcos, Lima, durante junio y julio de 2005. Se criaron 400 pollos bb de carne de un día de edad de la línea ROSS 308. La crianza fue por sexos mezclados. Las aves recibieron alimento comercial y agua $a d$ libitum, y se vacunaron contra la Enfermedad de Gumboro y el Newcastle, siguiendo el programa de rutina para pollos broiler.

Se utilizó viruta nueva de madera como material de cama. Esta fue tratada con un aluminosilicato comercial, que consiste en una bentonita cálcica cuya composición es $98.5 \%$ de montmorillonita, la cual ha sido activada con calor y ácido sulfúrico al 37\%. Las muestras de material de cama y aire para la determinación de amoniaco atmosférico se procesaron en los Laboratorios Sud-Chemie S.A., Callao.

\section{Diseño Experimental}

El tamaño de muestra para el estudio fue calculado utilizando la fórmula de diferencia de medias, tomando como parámetro el peso corporal, obteniéndose un tamaño de muestra de 41 animales como mínimo.

Los animales fueron separados en dos grupos de 200 aves cada uno, con 4 repeticiones de 50 aves por grupo y una densidad poblacional de 9 aves $/ \mathrm{m}^{2}$. El Grupo A, conformado por las aves alojadas sobre cama tratada con aluminosilicato, y el Grupo B, por las aves alojadas sobre cama sin tratar como control.

El tratamiento de la cama del grupo A se hizo tres días antes de la recepción de las aves, rociándose $0.55 \mathrm{~kg}$ de aluminosilicato $/ \mathrm{m}^{2} \mathrm{de}$ cama. Adicionalmente, el día 35 se realizó un segundo tratamiento a razón de $0.45 \mathrm{~kg} / \mathrm{m}^{2}$ de cama.

\section{Calidad de la Cama}

Niveles de amoniaco atmosférico. Se emplearon dos métodos:

a) Método de titulación por retroceso: Entre la $3^{\mathrm{a}}$ y la $6^{\mathrm{a}}$ semana de edad se tomaron muestras de $40 \mathrm{~L}$ de aire, con una frecuencia de dos veces por semana, a la altura de las fosas nasales de las aves. Se usó una bomba manual con una capacidad de $2 \mathrm{~L}$, haciendo burbujear el aire aspirado sobre $100 \mathrm{ml}$ de solución de ácido sulfúrico al $0.025 \mathrm{~N}$. Esta operación se repitió 20 veces hasta capturar los 40 litros de aire. E1 amoniaco presente en el aire entra en contacto con el ácido sulfúrico haciéndolo reaccionar, disminuyendo su concentración. El ácido restante se determinó por titulación con $\mathrm{NaOH} 0.025 \mathrm{~N}$, $\mathrm{y}$ por diferencia de concentraciones se obtuvo la cantidad de amoniaco presente en el aire muestreado.

b) Tarjetas Chromair System ${ }^{\circledR}$ de detección de amoniaco. Se realizaron 3 muestreos entre la $5^{\mathrm{a}}$ y $6^{\mathrm{a}}$ semana de edad. Las tarjetas se colocan a la altura de las fosas nasales de las aves $(20 \mathrm{~cm})$, durante un lapso de 2 minutos a 16 horas. Este dispositivo tiene una escala que se va coloreando de acuerdo a la concentración de amoniaco presente en el ambiente.

Medición del pH atmosférico y de la cama. La medición se hizo en ambos grupos y por cada repetición los días $0,7,14,18,21$, $25,28,32,35,39,42$ y 44 . Para el pH de la cama se colectó una muestra de $10 \mathrm{~g}$ de cama y se colocó en un matraz, adicionándose $500 \mathrm{ml}$ de agua destilada y agitándose por 10 minutos. Se dejó sedimentar los sólidos y se midió el pH de la solución directamente con un potenciómetro a temperatura ambiente. Para el ph atmosférico se colgaron cintas de papel indicador de $\mathrm{pH}$ por una semana. 
Medición de la humedad (\%) de la cama. Las muestras de cama de ambos grupos se tomaron los mismos días que para la medición del $\mathrm{pH}$. Se pesó de 10 a $15 \mathrm{~g}$ de cama en una placa petri y se llevó a la estufa a $110^{\circ} \mathrm{C}$ por 2 horas. Luego se colocaron en un desecador y se pesaron, y la humedad se determinó por diferencia entre los pesos.

\section{Lesiones en las Aves}

Se realizó al término del estudio (día 44) en 10 animales por repetición ( $n=40$ por grupo experimental).

Lesiones en la pechuga, en base a una escala del 0 al 4, donde el Grado 0 corresponde a una pechuga normal, y los grados 1, 2, 3 y 4 corresponden a costras, heridas o cambio de coloración menor a $1 \mathrm{~cm}$ en un 25, 50, 75 y $>75 \%$, respectivamente.

Lesiones en patas (La pododermatitis se calificó del 0 al 3 en base a una escala propuesta por Van der AA (2008):

Grado 0: Patas normales, ausencia de lesiones.

Grado 1: Erosiones muy leves en la almohadilla plantar y ligera coloración oscura.

Grado 2: Erosiones múltiples, heridas en la almohadillas plantar y dedos.

Grado 3: Erosiones severas, heridas profundas en la almohadilla plantar, dedos e hiperqueratosis.

\section{Parámetros Productivos}

En cada grupo experimental se pesó a cada animal en forma semanal. Se determinó el índice de conversión alimenticia (I.C.A) en base al alimento consumido $(\mathrm{kg})$ y el peso vivo producido $(\mathrm{kg})$. El índice de mortalidad se obtuvo en base a la mortalidad diaria y semanal de cada grupo; además, se realizó la necropsia para determinar la causa de las muertes. También se determinó el índice de eficiencia productivo europeo (I.E.P.E.) en base a la división del producto del cálculo porcentual de la viabilidad y la ganancia diaria de peso entre la eficiencia alimenticia y la edad de los pollos.

\section{Análisis de Datos}

El peso corporal, índice de conversión alimenticia, $\mathrm{pH}$ de cama, $\mathrm{pH}$ atmosférico y porcentaje de humedad de cama se evaluaron por la prueba de " $t$ " Student de independencia para determinar su relación. La presencia de lesiones en patas y pechuga fueron evaluados mediante la prueba de U de Mann Whitney. La mortalidad fue evaluada mediante la prueba de Chi-cuadrado. En todos los casos se empleó el programa STATA.

\section{Resultados}

Entre la primera y cuarta semana de vida se encontró mayor mortalidad en las aves criadas sobre cama no tratada $(2.5 \%, 5 / 200)$ en comparación con las aves en cama tratada $(1 \%, 2 / 200)$, aunque esta diferencia no fue estadísticamente significativa.

Los pollos del grupo no tratado (B) mostraron mayor peso corporal que los pollos del grupo tratado (A) durante toda la campaña; sin embargo, esta diferencia solo fue significativa en la semana 1 y $2(\mathrm{p}<0.05)$, y no así a los 44 días de edad, a pesar de mantener una diferencia de $55 \mathrm{~g}$ a favor del grupo $\mathrm{B}$ (Cuadro 1). Tampoco hubo diferencias estadísticas en el índice de conversión alimenticia (1.82 vs 1.83) ni en el índice de eficiencia productivo europeo (338.36 vs 338.07) entre el grupo tratado y no tratado, respectivamente.

No se presentaron ulceraciones en la zona de la pechuga y las lesiones halladas en las patas fueron similares para ambos grupos (Cuadro 2).

La concentración de amoniaco atmosférico no sobrepasó los 25 ppm (nivel máximo permisible para las aves) en ninguno de 
Cuadro 1. Peso corporal semanal (g) de pollos de carne criados sobre cama tratada con aluminosilicato vs cama no tratada

\begin{tabular}{lcccccccc}
\hline & \multicolumn{7}{c}{ Edad en días } \\
\cline { 2 - 9 } & 0 & 7 & 14 & 21 & 28 & 35 & 42 & 44 \\
\hline Tratada & 39 & $160^{\mathrm{a}}$ & $406^{\mathrm{a}}$ & 816 & 1,344 & 1,980 & 2,596 & 2,776 \\
No tratada & 40 & $167^{\mathrm{b}}$ & $425^{\mathrm{b}}$ & 833 & 1,363 & 2,004 & 2,645 & 2,831 \\
\hline
\end{tabular}

${ }^{\mathrm{a}, \mathrm{b}}$ Letras diferentes entre tratamientos dentro de una misma edad son estadísticamente diferentes $(p<0.05)$

Cuadro 2. Presencia de pododermatitis ${ }^{1}$ en pollos de carne criados hasta los 44 días de edad en cama tratada con aluminosilicato vs cama no tratada

\begin{tabular}{ccc}
\hline Grado de lesión & Cama tratada & Cama no tratada \\
\hline 0 & 16 & 15 \\
1 & 18 & 20 \\
2 & 6 & 5 \\
3 & 0 & 0 \\
\hline
\end{tabular}

${ }^{1}$ En base a Van der AA (2008)

los dos grupos durante toda la campaña; sin embargo, fue 10 ppm menor en el ambiente de las aves del grupo con cama tratada (Fig. 1). El $\mathrm{pH}$ de la cama y $\mathrm{pH}$ atmosférico fue estadísticamente menor durante casi toda la campaña en el grupo con aluminosilicato en relación con el grupo sin tratamiento en la cama $(\mathrm{p}<0.05)$ (Fig. 1).

El porcentaje de humedad de la cama se mantuvo constante en ambos grupos, incrementándose dramáticamente a partir del día 25 en ambos casos, sin encontrarse diferencia significativa (Fig. 1).

\section{Discusión}

Los tratamientos de cama enfocados a controlar la volatilización del amoníaco en los galpones han demostrado ser muy eficaces en la industria avícola y, actualmente, son ampliamente recomendados y adoptados por muchos productores (Lott y Donald, 2003); sin embargo, el tiempo que permanecen activos en la cama es relativamente corto. La mortalidad asociada a las emanaciones de gases de amoníaco se ve incrementada cuando estos alcanzan concentraciones mayores a 100 ppm (Reece y Lott, 1980). En el pre- 

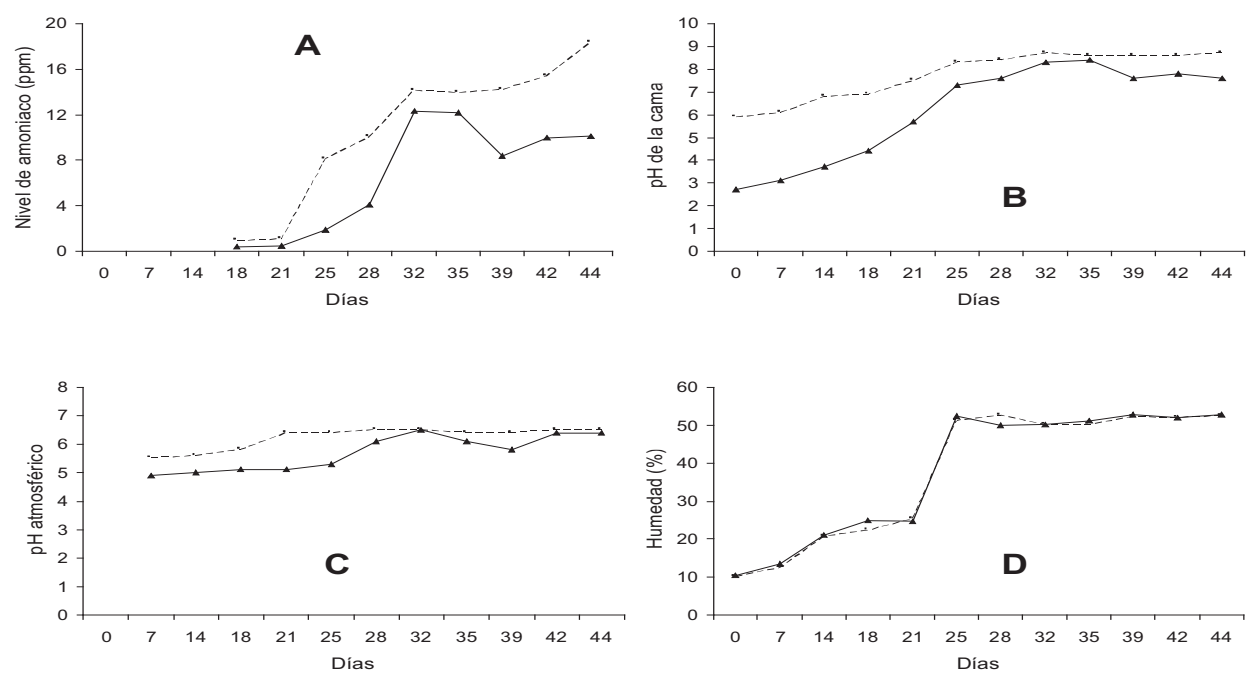

Figura 1. A) Niveles de amoniaco atmosférico (ppm), B) $\mathrm{pH}$ atmosférico, C) $\mathrm{pH}$ de la cama y D) porcentaje de humedad de la cama de pollos de carne criados sobre cama tratada con aluminosilicato

sente estudio no se llegó a estas concentraciones, de allí que la mortalidad observada en ambos grupos experimentales no fueron estadísticamente diferentes. Los niveles se mantuvieron por debajo de $25 \mathrm{ppm}$, límite sugerido por Reece et al. (1980) como rango normal establecido para una crianza comercial de pollos de carne. No obstante, las diferencias biológicas entre una tasa de mortalidad de $1.0 \%$ para pollos en cama tratada con aluminosilicato y $2.5 \%$ en el grupo control estuvo probablemente asociada a problemas relacionados a la mayor concentración de amoniaco en la cama no tratada, causando la muerte súbita, justificando el tratamiento efectuado a la cama.

Las aves del grupo control tuvieron un mayor peso en algunas semanas del estudio. Esta diferencia en la ganancia de peso y peso corporal final ocurrieron justamente después de cada uno de los dos tratamientos de la cama con el producto evaluado. Estas diferencias podrían estar relacionadas a un efecto depresor del aluminosilicato, dado a los hábitos de las aves de comer cama del gallinero. Además, se observó al momento de cada tratamiento la presencia de heces oscuras y diarreicas en el grupo tratado, no así en el grupo con cama no tratada.

Algunos investigadores (Benoff, 1982; Charles, 1982; Nascimento y Giovanni, 2002) sugieren que pollos expuestos a altos niveles de amoniaco asociado a una mala calidad de la cama tienen mayor probabilidad de desarrollar úlceras en la pechuga; sin embargo, otros se refieren a una mayor frecuencia a desarrollar ampollas pectorales en vez de úlceras (North y Bell, 1998) y un incremento de lesiones en las patas (Moum et al., 1969). En el presente trabajo no se observaron lesiones en la pechuga debido, probablemente, a que las concentraciones de amoniaco fueron bajas. Las lesiones observadas en las patas fueron ligeras y en frecuencia similar entre ambos grupos, y su presentación podría atribuirse a un aumento en la humedad de la cama de ambos grupos observado en las últimas semanas de crianza.

Los niveles de amoniaco atmosférico no sobrepasaron las $25 \mathrm{ppm}$ en ambos grupos; sin embargo, el nivel fue menor en el grupo 
de aves criadas sobre cama tratada. Al parecer, el aluminosililicato fue eficaz en neutralizar el amoniaco liberado de la cama durante las primeras cuatro semanas de crianza.

$\mathrm{El} \mathrm{pH}$ de la cama muestra una fuerte asociación con los niveles de amoníaco presente en los galpones. El pH de la cama fue menor en el grupo de aves criadas sobre cama tratada en comparación al grupo no tratado mientras duró el efecto del aluminosilicato, llegando a ser similares al día 35 . Valores de $\mathrm{pH}$ en la cama por encima de 8.0 ocasionan una mayor concentración de amoniaco atmosférico, debido al crecimiento de microorganismos con capacidad de descomponer el ácido úrico para la formación de amoniaco (Blake y Hess, 2001); también un pH alcalino favorece a la uricasa a catalizar la descomposición del ácido úrico. Por otro lado, los valores de $\mathrm{pH}$ de cama menores a 7.0 obtenidos por el grupo de cama tratada se debieron al efecto acidificante del aluminosilicato sobre la misma mientras estuvo activo el producto. Al parecer, el $\mathrm{pH}$ de la cama se ve afectado con el aumento del porcentaje de humedad de la cama, haciéndose más alcalina a valores más altos de humedad.

El pH atmosférico fue mayor en el grupo de aves criadas sobre cama no tratada comparados a las aves criadas sobre cama tratada. El pH atmosférico es un indicador de la calidad del aire. En este estudio se pudo observar que las variaciones en los valores de $\mathrm{pH}$ se encuentran ampliamente relacionadas a los niveles de amoniaco atmosférico que presentaron ambos grupos.

No se encontró diferencias entre grupos con relación al porcentaje de humedad de la cama. La humedad permaneció dentro del rango óptimo (25 a 35\%) durante las primeras cuatro semanas del estudio; sin embargo, mostró un elevado incremento a partir del día 25, llegando a valores superiores al $50 \%$ hasta el fin del estudio. Este incremento en la humedad de la cama pudo deberse a un deficiente manejo de la ventilación y al uso de bebederos tipo canaleta empleados en las últimas semanas de crianza; además, las aves aportan mayor humedad al ambiente a medida que van creciendo (Donald, 1997). La estación de invierno en la que fueron criadas las aves también pudo haber influido en el contenido de la humedad de la cama. Este aumento del porcentaje de humedad de la cama favoreció el incremento de los niveles de amoniaco, incluyendo en los animales con cama tratada (Wyatt, 1985).

\section{Conclusiones}

- El tratamiento de la cama con aluminosilicato fue eficaz en controlar el amoniaco, llegando a obtenerse una menor concentración de amoniaco en la cama entre la tercera a la sexta semana de edad.

\section{LiTERATURA CitTADA}

1. Al-Homidan A, Robertson JF, Petchey AM. 2003. Review of the effect of ammonia and dust concentrations on broiler performance. World Poultry Sci J 59: 340-349.

2. Benoff FH. 1982. Vigile niveles de amoníaco en el pabellón de pollos asaderos. Rev Ind Avícola 29(5): 32.

3. Blake P, Hess J. 2001. Poultry Guard ${ }^{\mathrm{TM}}$ as a litter amendment. Auburn University. Aces publications: Anr-1209. [Internet]. Available in: http://Aces \%20 Publications \%20\%20ANR-1209.htm

4. Carlile FS. 1984. Ammonia in poultry houses: Review. World Poultry Sci J 40: 99-113.

5. Charles H. 1982. Factores que afectan las úlceras en la pechuga en pollos de asar. Rev Ind Avícola 29(5): 42.

6. Donald J. 1997. Tendencias en el control ambiental en galpones avícolas. Rev Ind Avícola 44(6): 10-17.

7. Jodas S, Hafez HM. 2001. Manejo de la cama y enfermedades relacionadas de los pavos. Rev Avicultura Profesional 19(5): 17-21. 
8. Lott B, Donald J. 2003. Amônia. Grandes perdas mesmo quando você não percebe. Catálogo Oficial Da Ave Sui. Rev Aviculura Industrial 94(4): 34-35.

9. Moum SG, Seltzer W, Goldhaft TM. 1969. A simple method of determining concentrations of ammonia in animal quarters. Poultry Sci 48: 347-348.

10. Nascimento VM, Giovanni P. 2002. Qualidade de carcaça e o manejo na produção. Rev Avicultura Industrial 93(5): 12-16.

11. North MO, Bell D. 1998. Manual de producción avícola. $3^{a}$ ed. México: Manual Moderno. $175 \mathrm{p}$.

12. Quintana J. 1999. Avitecnia: Manejo de las aves domésticas más comunes. $3^{\mathrm{a}}$ ed. México: Ed Trillas. 384 p.
13. Reece FN, Lott BD. 1980. The effect of ammonia and carbon dioxide during brooding on the performance of broiler chickens. Poultry Sci 59: 1664-1661.

14. Reece FN, Lott BD, Deaton JW. 1980. Ammonia in the atmosphered during brooding affects performance of broiler chick. Poultry Sci 59: 486.

15. Sainsbury D. 2000. Poultry health and management - chickens, turkeys, ducks, geese, and quail. $4^{\text {th }}$ ed. Oxford, UK: Blackwell Scientific. 204 p.

16. Van $\operatorname{der} A a A$. 2008. Clay minerals to fight footpad lesions. World Poultry 24: 15-17.

17. Wyatt R. 1985. La ventilación en los galpones. Rev Avicultura Profesional 3(2): 75-76. 